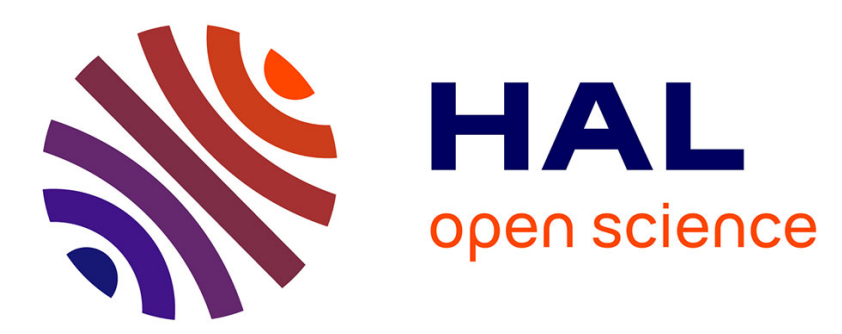

\title{
Nonlinear Power Amplifier Characteristic Based on Orthogonal Polynomials Method for FBMC Systems
}

Ditthawat Songratthaset, Suwat Pattaramalai

\section{To cite this version:}

Ditthawat Songratthaset, Suwat Pattaramalai. Nonlinear Power Amplifier Characteristic Based on Orthogonal Polynomials Method for FBMC Systems. 11th IFIP Wireless and Mobile Networking Conference (WMNC 2018), Sep 2018, Prague, Czech Republic. pp.67-72. hal-01995434

\section{HAL Id: hal-01995434 \\ https://hal.inria.fr/hal-01995434}

Submitted on 26 Jan 2019

HAL is a multi-disciplinary open access archive for the deposit and dissemination of scientific research documents, whether they are published or not. The documents may come from teaching and research institutions in France or abroad, or from public or private research centers.
L'archive ouverte pluridisciplinaire HAL, est destinée au dépôt et à la diffusion de documents scientifiques de niveau recherche, publiés ou non, émanant des établissements d'enseignement et de recherche français ou étrangers, des laboratoires publics ou privés.

\section{(c)(1)}

Distributed under a Creative Commons Attribution| 4.0 International License 


\section{Nonlinear Power Amplifier Characteristic Based on Orthogonal Polynomials Method for FBMC Systems}

\author{
Ditthawat Songratthaset \\ Electrical and Telecommunication Engineering Department \\ King Mongkut's University of Technology Thonburi \\ Bangkok, Thailand \\ ditthawat.s@mail.kmutt.ac.th
}

\author{
Suwat Pattaramalai \\ Electrical and Telecommunication Engineering Department \\ King Mongkut's University of Technology Thonburi \\ Bangkok, Thailand \\ suwat.pat@kmutt.ac.th
}

\begin{abstract}
In this paper, an analytical expression of nonlinear power amplifier characteristic based on orthogonal polynomials method for filter bank multicarrier modulation (FBMC) systems is proposed. FBMC has been widely deployed for broadband wireless communications. Nevertheless, one of the drawbacks of the FBMC systems is a large peak-to-average power ratio (PAPR) in its time domain signal. This leads to a nonlinear distortion when the signal is passed through a high power amplifier (HPA) of a transmitter. In this paper, an analytical computation of the HPA characteristic based on the orthogonal polynomial method for baseband uniform, exponential, and Rayleigh input distribution with odd and even polynomial coefficient order is proposed. The HPA modelling error comparison between the proposed orthogonal method and the conventional polynomial method is investigated at a different IBO. Simulation results show that the proposed orthogonal polynomial method is superior to the conventional polynomial method. Moreover, the HPA modelling error of the proposed orthogonal polynomial method for uniform input distribution yield better result compared with the exponential and the Rayleigh input distribution case.
\end{abstract}

Keywords-HPA; FBMC; orthogonal polynomial; nonlinear power amplifier

\section{INTRODUCTION}

It is well-known that the filter bank multicarrier modulation (FBMC) technique, which is the multicarrier techniques-based communication, will play an important solution for achieving high data rate $5 \mathrm{G}$ communications [1]. However, a major drawback of the FBMC signal is the high Peak to Average Power Ratio (PAPR), which arise from the superposition of many variations in the signal envelope. When the signal is passed through a nonlinear device such as a high power amplifier (HPA), a significant nonlinear distortion is occurred, which lead to a performance degradation of the system. To avoid the distortion of the signal, the HPA linearization design is often required. Many HPA linearization methods have been proposed based on the look-up table (LUT) or models such as a Volterra series, the rational function, Wiener-Hammerstein systems, and memory polynomial model [2]. For model-based approaches, the polynomial model is the simplest technique and is commonly used for implementation. Nevertheless, there is a lack of study on the analytical computation of the HPA characteristic. In [3], a performance analysis for bit error rate expression is derived for FBMC/OQAM system with non-linear HPA by varying constellation size in OQAM modulation and input back-off. In [4]. the impact of the nonlinear distortions and a theoretical characterization of a soft envelope limiter (SEL) HPA model in the orthogonal frequency division multiplex (OFDM) systems has been investigated. However, an analytical HPA characteristic expression proposed in [4] could not be easily applied to the solid state power amplifier (SSPA), and the travelling wave tube amplifier (TWTA) HPA model. In [5], a complete theoretical characterization of the nonlinear HPA for FBMC systems has been proposed by using a polynomial fitting method, which can be utilized to any type of HPA models. In [6], the effect of the nonlinear based on memory-less HPA models have been studied and an analysis BER performance of FBMC schemes based on the polynomial approximation method have been proposed. There are researches regarding the analytical HPA characteristic expression based on the polynomial mathematic method. Nevertheless, the basic polynomial approximation could not be well suited comparing with other mathematic method [7]. This fact motivates us to propose an analytical expression of nonlinear power amplifier characteristic based on the orthogonal polynomial method for FBMC system. Moreover, the HPA characterization in a different input distribution is further investigated in order to ensure the numerical efficiency of the orthogonal polynomials method. The HPA modelling error, verified by a normalized mean squared error (NMSE), comparing between the conventional polynomial method and the proposed orthogonal polynomial method at a several IBO values is determined. The contributions of this paper is to derive the HPA characteristic expression by using the orthogonal polynomial mathematic method with odd and even coefficient order terms in three input distribution cases, which are 1) the uniform based-orthogonal polynomial method, 2) the exponential based-orthogonal polynomial method, 3) the Rayleigh based-orthogonal polynomial method.

The organization of this paper is as follows. In section II the HPA models characteristic of the FBMC systems and the nonlinear HPA conversion characteristics are described. In Section III, we detail the derivation of the proposed orthogonal polynomial method. The simulation results and the conclusion are given in Section IV and V, respectively. 


\section{SYSTEM MODEL}

In this section, the HPA models characteristic of the FBMC systems and the nonlinear HPA conversion based on the polynomial method are described.

\section{A. HPA Model}

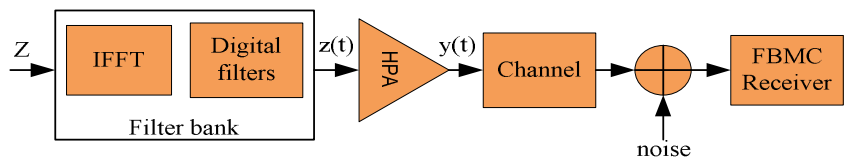

Fig. 1. FBMC systems.

Figure 1 shows the FBMC systems block scheme. The modulated symbol $Z$ in the frequency domain is converted to the time domain signal by the IFFT and digital filters processing for generating an FBMC signal $z(t)$. After that, the baseband signals is passed through a nonlinear high power amplifier (HPA), which leads to a substantial amount of signal distortion. The amplified signal $y(t)$ is defined by the AM/AM characteristic $\left(F_{a}(\rho)\right)$ and the $\mathrm{AM} / \mathrm{PM}$ characteristic $\left(F_{p}(\rho)\right)$ of the HPA as [4]

$$
\begin{gathered}
y(t)=F_{a}(\rho) \exp \left(j F_{p}(\rho)\right) \exp (j \theta) \\
=S(\rho) \exp (j \theta)
\end{gathered}
$$

where $\rho, \theta$ is the signal input modulus and phase respectively. $S(\rho)=F_{a}(\rho) \exp \left(j F_{p}(\rho)\right)$ is the complex soft envelop of the amplified signal $y(t)$.

According to the Bussgang theorem [8], the nonlinear HPA output $y(t)$ can be written as

$$
y(t)=K(t) z(t)+d(t)
$$

where $K(t)$ is a complex gain and $d(t)$ is an additive zero mean noise uncorrelated from $z(t)$. For the sake of simplicity, the time variable $(t)$ is discarded.

The complex gain $K$ can be determined by

$$
K=\frac{1}{2} \mathbb{E}\left[\frac{\partial S(\rho)}{\partial \rho}+\frac{S(\rho)}{\rho}\right]
$$

where $\mathbb{E}[$.$] is the expectation operator.$

The variance $\sigma_{d}^{2}$ of the nonlinear distortion noise $d(t)$ can be determined by evaluating its mean [4]. From (2), $\sigma_{d}^{2}$ is given by

$$
\begin{aligned}
\sigma_{d}^{2}= & \mathbb{E}\left[|d(t)|^{2}\right] \\
& =\mathbb{E}\left[|y(t)|^{2}\right]-|K|^{2} \mathbb{E}\left[\rho^{2}\right]
\end{aligned}
$$

Substituting $y(t)$ in (1) into (4), the variance $\sigma_{d}^{2}$ is

$$
\sigma_{d}^{2}=\mathbb{E}\left[|s(\rho)|^{2}\right]-|K|^{2} \mathbb{E}\left[\rho^{2}\right]
$$

In general, the HPA is operated at a given input back-off (IBO) from a given level for reducing the effects of nonlinearities. The IBO is defined as

$$
I B O=10 \log _{10}\left(\frac{A_{\text {sat }}^{2}}{\sigma^{2}}\right)
$$

where $\sigma$ is the variance of the input signal (mean input signal power) and $A_{\text {sat }}$ is the HPA input saturation level.

Basically, a normalized mean squared error (NMSE) is employed in order to verify the quantitative measure HPA modelling error defined as [9]

$$
n m s e(d B)=10 \log _{10}\left[\frac{\sum_{n=1}^{D}\left|y\left(t_{n}\right)-\hat{y}\left(t_{n}\right)\right|^{2}}{\sum_{n=1}^{N}\left|y\left(t_{n}\right)\right|^{2}}\right]
$$

where $y\left(t_{n}\right)$ is the measured HPA output and $\hat{y}\left(t_{n}\right)$ is the HPA modelled output. $D$ is the number of input samples.

\section{B. The Nonlinear HPA Characterization Based on the Polynomial Method}

Based on a nonlinear power amplifier system, the baseband output $y(t)$ of a polynomial model can be written as [10]

$$
y(t)=\sum_{k=1}^{L} a_{k} \emptyset_{k}(z(t))
$$

where $\emptyset_{k}(z(t))=|z(t)|^{k-1} z(t)$ is the conventional polynomial basis function. $z(t)$ is the HPA input and $L$ is the polynomial order. $a_{k}$ is the conventional polynomial coefficient at order $k$, which can be calculated by using the least-squares (LS) solution [9].

In order to simplify the computation and achieve the analytical expressions of the complex gain $(K)$, the new HPA output $y(t)$ is written as [5] 


$$
\begin{aligned}
y(t) & =e^{j \theta} \sum_{k=1}^{L} a_{k} \rho^{k} \\
& =e^{j \theta} S(\rho)
\end{aligned}
$$

Therefore, the polynomial approximation of $S(\rho)$ is

$$
S(\rho)=\sum_{k=1}^{L} a_{k} \rho^{k}
$$

In order to determine the complex gain $(K),(10)$ is substituted into (3) and the expression is derived to obtain the following equation

$$
\begin{aligned}
K= & \frac{1}{2} \mathbb{E}\left[\frac{\partial}{\partial \rho}\left(\sum_{k=1}^{L} a_{k} \rho^{k}\right)+\left(\frac{\sum_{k=1}^{L} a_{k} \rho^{k}}{\rho}\right)\right] \\
= & \frac{1}{2} \mathbb{E}\left[\left(\sum_{k=1}^{L} a_{k} k \rho^{k-1}\right)+\left(\sum_{k=1}^{L} a_{k} \rho^{k-1}\right)\right] \\
& =\frac{1}{2} \sum_{k=1}^{L} a_{k}(k+1) \mathbb{E}\left(\rho^{k-1}\right)
\end{aligned}
$$

The expectation of a power of the Rayleigh random variable is investigated by the $k$ th-order derivation of the moment-generating function (MGF). Finally, the analytical expressions of the complex gain $(K)$ for any HPA model based on the polynomial method can be defined by [5]

$$
\begin{aligned}
K & =\sqrt{\frac{\pi}{8}} \sum_{k=1, k \text { odd }}^{L}(k+1) a_{k} \sigma^{k} \prod_{i=0}^{\frac{k-1}{2}}(2 i+1) \\
& +\frac{1}{2} \sum_{k=2, k \text { even }}^{L}(k+1) a_{k}(\sqrt{2} \sigma)^{k}\left(\frac{k}{2}\right) !
\end{aligned}
$$

\section{THE PROPOSED METHOD}

The nonlinear HPA is an important section in the communication system because its nonlinearity causes a substantial amount of signal distortion. Therefore, an analytical HPA characterization is often necessary issue. In this paper, an orthogonal polynomial fitting method for the HPA characterization is proposed for uniform, exponential and Rayleigh input distribution case.

\section{A. The Uniform Based-Orthogonal Polynomial Method}

Based on the conventional polynomial method, the numerical instability is one of the major problems. To cope with such issue, the orthogonal basis function is considered by using the orthogonal polynomial basis function.

The HPA output $y(t)$ based on the orthogonal polynomial method is modified as

$$
y(t)=\sum_{k=1}^{L} \mu_{k} \varphi_{k}(z(t))
$$

where $\mu_{k}$ is the orthogonal polynomial coefficient at order $k$, which can be calculated by using the least-squares (LS) solution [9]. $L$ is the polynomial order and $\varphi_{k}$ is the orthogonal polynomial basis function with odd and even polynomial coefficient order $k$.

To obtain the closed-form expressions for the orthogonal polynomials, an upper triangular matrix $U$ cloud be applied. The orthogonal polynomial basis function can be examined by

$$
\varphi_{k}(z(t))=\sum_{m=1}^{k} U_{k m}|z(t)|^{m-1} z(t)
$$

where $U_{k m}$ is an upper triangular matrix with $k$ th-order orthogonal polynomial for the uniformly distribution given by

$$
U_{k m}=(-1)^{k+m} \frac{(k+m) !}{(m-1) !(m+1) !(m-k) !}
$$

The baseband output $y(t)$ in (13) can be modified as

$$
\begin{aligned}
y(t) & =\sum_{k=1}^{L} \mu_{k} \sum_{m=1}^{k} U_{k m}|Z(t)|^{m-1} Z(t) \\
& =e^{j \theta(t)}\left[\sum_{k=1}^{L} \mu_{k} \sum_{m=1}^{k} U_{k m} \rho^{m}\right]
\end{aligned}
$$

Therefore, the orthogonal polynomial expression of $S(\rho)$ is

$$
S(\rho)=\sum_{k=1}^{L} \mu_{k} \sum_{m=1}^{k} U_{k m} \rho^{m}
$$


The HPA gain based on the orthogonal polynomial method for the uniformly distribution can be determined by substituting (17) into (3), which is

$$
\begin{gathered}
K=\frac{1}{2} \mathbb{E}\left[\frac{\partial}{\partial \rho}\left(\sum_{k=1}^{L} \mu_{k} \sum_{m=1}^{k} U_{k m} \rho^{m}\right)+\left(\frac{\sum_{k=1}^{L} \mu_{k} \sum_{m=1}^{k} U_{k m} \rho^{m}}{\rho}\right)\right] \\
K=\frac{1}{2} \mathbb{E}\left[\sum_{k=1}^{L} \mu_{k} \sum_{m=1}^{k} U_{k m}\left(m \rho^{m-1}\right)+\sum_{k=1}^{L} \mu_{k} \sum_{m=1}^{k} U_{k m}\left(\rho^{m-1}\right)\right] \\
K=\frac{1}{2} \sum_{k=1}^{L} \mu_{k} \sum_{m=1}^{k} U_{k m} \mathbb{E}\left[\left(m \rho^{m-1}\right)+\left(\rho^{m-1}\right)\right] \\
K=\frac{1}{2} \sum_{k=1}^{L} \mu_{k} \sum_{m=1}^{k} U_{k m}(m+1) \mathbb{E}\left[\left(\rho^{m-1}\right)\right]
\end{gathered}
$$

\section{B. The Exponential Based-Orthogonal Polynomial Method}

In theory, the orthogonal polynomial basis is related to the probability density functions (PDF) of the input signal. The PDF, denoted as $f_{\rho}(\rho)$, for the truncated exponential input distribution is defined by [9]

$$
f_{\rho}(\rho)=\frac{1}{1-e^{-(1 / \lambda)}} \frac{1}{\lambda} e^{-(\rho / \lambda)}, 0 \leq \rho \leq 1
$$

when $\lambda$ is the variance of the input signal and $\rho$ is related to the input signal. Basically, the expectation of random variable signals can be determined from its PDF.

The moment-generating function (MGF) of the truncated exponential distribution can be defined by

$$
M_{\rho}(t)=\frac{\int_{0}^{1} e^{-t \rho} \cdot \rho f(\rho) d \rho}{1-e^{-(1 / \lambda)}}
$$

Therefore, the expectation of the truncated exponential distribution is investigated by the $m$ th-order moment, which is [11]

$$
\mathbb{E}\left[\rho^{m}\right]=\frac{m ! \lambda^{m}}{1-e^{-(1 / \lambda)}}
$$

Substituting (21) into (18), the complex gain $K$ for the exponential input distribution is given by

$$
K=\frac{1}{2} \sum_{k=1}^{L} \mu_{k} \sum_{m=1}^{k} U_{k m}(m+1) \frac{m ! \lambda^{m}}{1-e^{-(1 / \lambda)}}
$$

\section{The Rayleigh Based-Orthogonal Polynomial Method}

The PDF of the truncated Rayleigh input distribution is determined by

$$
f_{\rho}(\rho)=\frac{1}{1-e^{-\left(1 / 2 \sigma^{2}\right)}} \frac{\rho}{\sigma^{2}} e^{-\left(\rho^{2} / 2 \sigma^{2}\right)}, 0 \leq \rho \leq 1
$$

The moment-generating function (MGF) of the truncated Rayleigh distribution can be defined by

$$
M_{\rho}(t)=\frac{\int_{0}^{1} e^{-t \rho} \cdot \rho f(\rho) d \rho}{1-e^{-\left(1 / 2 \sigma^{2}\right)}}
$$

The expectation of the truncated Rayleigh distribution is investigated by the $m$ th-order moment, which is [11]

$$
\mathbb{E}\left[\rho^{m}\right]=\frac{(\sqrt{2} \sigma)^{m}(m / 2) !}{1-e^{-\left(1 / 2 \sigma^{2}\right)}}+\frac{\sqrt{\pi / 2} \sigma^{m} 1 \cdot 3 \cdot 5 \ldots m}{1-e^{-\left(1 / 2 \sigma^{2}\right)}}
$$

Similarly, the complex gain $K$ is examined by substituting (25) into (18). The HPA gain based on the orthogonal polynomial method for the Rayleigh distribution is expressed as

$$
\begin{aligned}
K=\frac{1}{2} \sum_{k=1}^{L} \mu_{k} & {\left[\sum_{m=2, m \text { even }}^{k} U_{k m}(m+1)\left(\frac{(\sqrt{2} \sigma)^{m}(m / 2) !}{1-e^{-\left(1 / 2 \sigma^{2}\right)}}\right)\right.} \\
& \left.+\sum_{m=1, \text { modd }}^{k} U_{k m}(m+1)\left(\frac{\sqrt{\pi / 2} \sigma^{m} 1 \cdot 3 \cdot 5 \ldots m}{1-e^{-\left(1 / 2 \sigma^{2}\right)}}\right)\right]
\end{aligned}
$$

\section{Simulation RESUlTS}

In this section, based on a computer simulation, the performance of the derived HPA characterization is evaluated in terms of a HPA modelling error, which is verified by a normalized mean squared error (NMSE). Without loss of generality, the noise distortion parameter is not considered in the HPA characteristic expression. In our analysis, the commonly used SSPA model is employed as the HPA output $\hat{y}\left(t_{n}\right)$ for investigating the NMSE with parameters $A_{\text {sat }}=1, v$ $=10$ [12]. In the simulations, a total of 5000 input and output 


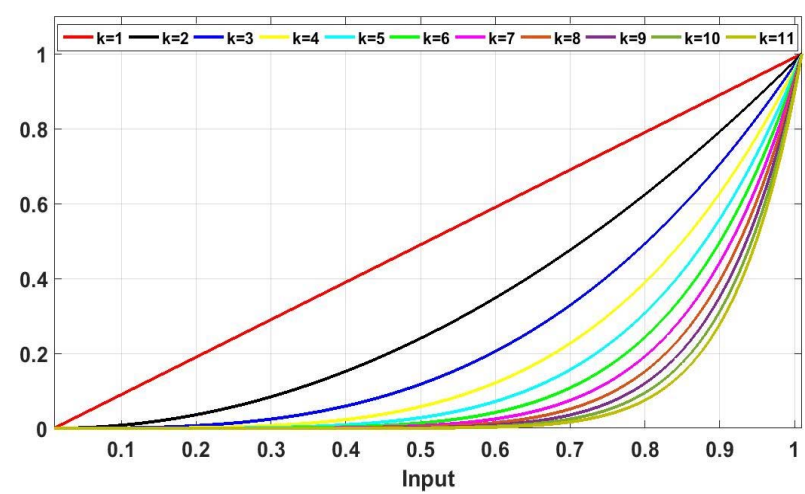

Fig. 2. The conventional polynomial basis.

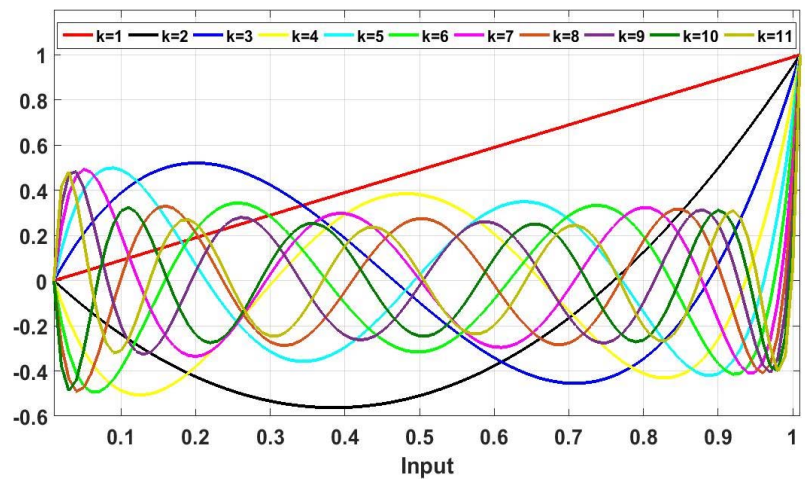

Fig. 3. The orthogonal polynomial basis.

samples are available. The conventional polynomial basis function with the different polynomial order $k$ is illustrated in Figure 2 and the orthogonal polynomial basis function is shown in Figure 3.

Figure 4, 5 and 6 presents the HPA modelling error comparison between the conventional polynomial method and the orthogonal polynomial method including the uniform based-orthogonal polynomial method, the exponential basedorthogonal polynomial method and the Rayleigh basedorthogonal polynomial method with different polynomial order $k$ at $\mathrm{IBO}=4,6,8$, respectively. In Figure 4 at the polynomials order 1, the HPA modelling error of the uniform, the exponential and the Rayleigh based-orthogonal polynomial method is equal to $-4.5 \mathrm{~dB},-0.3 \mathrm{~dB}$ and $-1 \mathrm{~dB}$, respectively. At the polynomial order 11, the HPA modelling error of the uniform, the exponential and the Rayleigh based-orthogonal polynomial method is equal to $-10 \mathrm{~dB},-6.8 \mathrm{~dB}$ and $-6 \mathrm{~dB}$, respectively. For the conventional polynomial method, the HPA modelling error is equal to $0 \mathrm{~dB}$ and $-1.9 \mathrm{~dB}$ at polynomial order 1 and 11 , respectively. From the figure, it clearly seen that the HPA modelling error of the orthogonal polynomial method is yield the good performance compared with the conventional polynomial method for full polynomial order ranges . However, the exponential and Rayleigh basedorthogonal polynomial method outperforms the conventional polynomial method largely when the order is more than 10 .

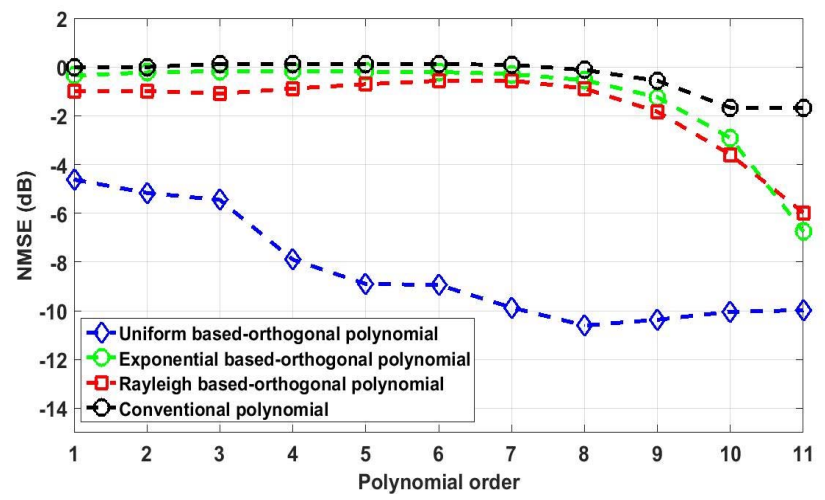

Fig. 4. The HPA modelling error comparison at $\mathrm{IBO}=4$

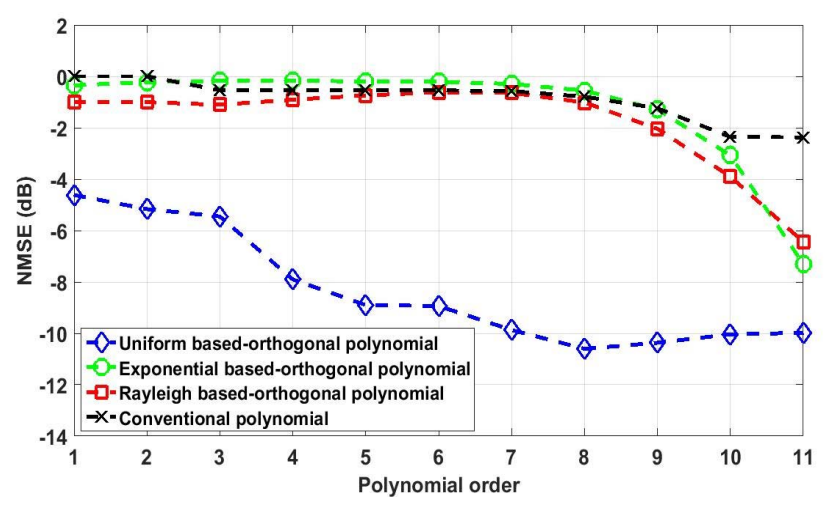

Fig. 5. The HPA modelling error comparison at $\mathrm{IBO}=6$

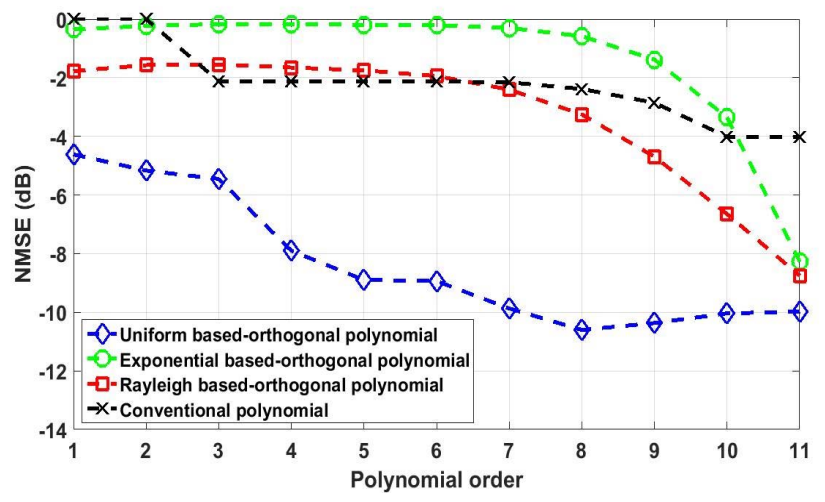

Fig. 6. The HPA modelling error comparison at $\mathrm{IBO}=8$

As a result, it is implied that the orthogonal polynomial method offer the good performance when a high order is applied. From Figure 5, it could be observed that the uniform based-orthogonal polynomial method offers the same HPA modelling error. Considered the polynomial order 11 at IBO 6, the HPA modelling error of the conventional polynomial method, the exponential and the Rayleigh based-orthogonal polynomial method is equal to $-2.2 \mathrm{~dB},-7.5 \mathrm{~dB}$ and $-6.5 \mathrm{~dB}$, which is less than that of IBO 4 by $0.3 \mathrm{~dB}, 0.7 \mathrm{~dB}$ and $0.5 \mathrm{~dB}$, respectively. At polynomial order 11 at IBO 8, the HPA modelling error of the uniform, the exponential and the 
Rayleigh based-orthogonal polynomial method is equal to -10 $\mathrm{dB},-8.3 \mathrm{~dB}$ and $-8.8 \mathrm{~dB}$, which yield the better result comparing with the conventional polynomial as illustrated in Figure 6. However, the exponential based-orthogonal polynomial method is better than the conventional polynomial method when the order is more than 10. As a result, it is clearly seen that the uniform based-orthogonal polynomial method provides the lowest HPA modelling error. Moreover, it is worth noticing that the exponential and the Rayleigh based-orthogonal polynomial method provide higher HPA modelling error when IBO value increase, meanwhile, the uniform based-orthogonal polynomial method do not introduce a different result for any IBO values.

For comparing among the three methods based on the orthogonal polynomial scheme, it could be found that the uniform based-orthogonal polynomial method outperforms the exponential and the Rayleigh based-orthogonal polynomial method, especially at a low IBO value.

\section{CONCLUSIONS}

In this paper, an analytical derivation of the HPA characteristic expression by using the orthogonal polynomial fitting method for baseband uniform, exponential, and Rayleigh input distribution with odd and even polynomial coefficient order is proposed. From the simulation results, it is clearly seen that the orthogonal polynomial methods outperform the conventional polynomial method, especially when a high order is applied. Among three methods based on the orthogonal polynomial scheme, the uniform basedorthogonal polynomial method introduces less error comparing with the exponential and the Rayleigh basedorthogonal polynomial method for full polynomial order ranges. Moreover, the uniform based-orthogonal polynomial methods do not introduce a different result for any IBO values, in contrast with the exponential and the Rayleigh basedorthogonal polynomial method. As a result, it could be implied that the nonlinear distortion caused by the HPA is more improved by using the proposed model, which could enhance the BER performance of the systems. For the future work, the BER performance analysis is investigated.

\section{REFERENCES}

[1] B. F. Boroujeny, "Filter bank multicarrier modulation: a waveform candidate for 5G and beyond," Hindawi journal on Advances in Electrical Engineering, vol. 2014, December 2014, pp. 1-25.

[2] R. Zayani et al, "Adaptive predistortion techniques for non-linearly amplified FBMC-OQAM signals", Proceeding of IEEE International Conference on Vehicular Technology (VTC Spring), Seoul, May 2014, pp. 1-5.

[3] G. U. Maheswari, A. Govindasamy, and S. J. Thiruvengadam, "Performance analysis of filter bank multicarrier system with non-linear high power amplifiers for 5G wireless networks", IET Signal Processing, vol. 11, no. 1, February 2017, pp. 66-72.

[4] D. Dardari, V. Tralli, and A. Vaccari, "A theoretical characterization of nonlinear distortion effects in OFDM systems", IEEE Trans on Communications, vol. 48, no. 10, October 2000, pp. 1755-1764.

[5] H. Bouhadda et al. "Theoretical analysis of BER performance of nonlinearly amplified FBMC/OQAM and OFDM signals", EURASIP Journal on Advances in Signal Processing, DOI: 10.1186/1687-61802014-60, May 2014.

[6] G. U. Maheswari, et al, "Performance analysis of filter bank multicarrier system with non-linear high power amplifiers for $5 \mathrm{G}$ wireless network," IET Journal on Advances in Signal Processing, DOI: 10.1049/ietspr.2015.0226, September 2016, pp. 66-72.

[7] R. Dallinger et al., "Adaptive pre-distortion techniques based on orthogonal polynomials signals", Proceeding of IEEE International Asilomar Conference on Systems and Computers (ASILOMAR), Canada, November 2010, pp. 1945-1950.

[8] A. PapoulisHe Rowe, Memoryless Nonlinearity with Gaussian Inputs : Elementary Results, Bell Syst. Tech. J. vol.61, no.7, 1982, pp. 15191526.

[9] R. Raich et al, "Orthogonal polynomials for power Amplifier modeling and predistorter design," IEEE Trans. on Vehicular Technology, vol. 53, no. 5, September 2004, pp. 1468-2797.

[10] T. Cunha, E. Lima and J. Pedro, "Validation and physical interpretation of the power-amplifier polar Volterra model," IEEE Trans. On Microw. Theory Tech., vol. 58, no. 12, December 2010, pp. 4012-4021.

[11] C. Walck, Statistical Distribution for Experimentalists Handbook. University of Stockholm, 2007.

[12] C. Rapp, "Effect of HPA nonlinearity on 4-DPSK-OFDM signal for digital sound broadcasting systems," Second European Conference on Sat. Comm, Belgium, 1997. 